\title{
FLAP Collaboration: Tasks and Perspectives. Study of Fundamentals and New Applications of Controllable Generation of Electromagnetic Radiation by Relativistic Electrons Using Functional Materials
}

A. Baldin ${ }^{a}$, A. Aryshev ${ }^{i, j}$, A. Avetisyan ${ }^{l}$, L. Aloyan $l$, E. Baldina ${ }^{a, *}$, Yu. Bazarov $^{g}$, D. Bogoslovsky ${ }^{a}$, V. Bleko ${ }^{a}$, A. Beloborodov ${ }^{a}$, A. Bulavskaya ${ }^{c}, Y_{\text {Yu. Cherepennikov }}^{c}$, Y. Dalyan ${ }^{l}$, A. Dorokhov ${ }^{a}$, A. Fedorov ${ }^{a}$, K. Fedorov $f$, M. Gostkin ${ }^{a}$, L. Grigoryan ${ }^{k}$, N. Karapetyan ${ }^{l}$, P. Karataev $f$, M. Karpov ${ }^{g}$, P. Kharyuzov ${ }^{a}$, V. Kocharyan ${ }^{k}$, D. Korovkin ${ }^{a}$, S. Kuleshov ${ }^{h}$, A. Safonov ${ }^{a}$, I. Kishchin ${ }^{b}$, V. Kim ${ }^{d, e}$, V. Kobets ${ }^{a}$, A. Kubankin ${ }^{b}$, E. Kuznetsova ${ }^{d, e}$, A. Mkrtchyan $^{k}$, A. Movsisyan ${ }^{k}$, L. Myshelovka ${ }^{b}$, R. Nazhmudinov ${ }^{b}$, K. Popov ${ }^{j}$ A. Potylitsyn ${ }^{c}$, A. Saharian ${ }^{k}$, I. Samofalova ${ }^{a}$, A. Shahbazyan ${ }^{l}$, S. Stuchebrov ${ }^{c}$, A. Trifonov ${ }^{a}$, S. Tyutyunnikov ${ }^{a}$, K. Vokhmyanina ${ }^{b}$, K. Yunenko ${ }^{a}$, V. Zakhvalinskii ${ }^{b}$, J. Zamora Saa ${ }^{h}$, A. Zelenov ${ }^{d, e}$, and A. Zhemchugov ${ }^{a}$

${ }^{a}$ Joint Institute for Nuclear Research, Dubna, 141980 Russia

${ }^{b}$ Belgorod National Research University, Belgorod, 308015 Russia

${ }^{c}$ National Research Tomsk Polytechnic University, Tomsk, 634050 Russia

${ }^{d}$ Petersburg Nuclear Physics Institute, National Research Center "Kurchatov Institute," Gatchina, 188300 Russia

e St. Petersburg Polytechnic University, St. Petersburg, 195251 Russia

${ }^{f}$ John Adams Institute at Royal Holloway, University of London, Egham, Surrey, TW20 OEX, UK

${ }^{g}$ Federal State Unitary Enterprise Russian Federal Nuclear Center - All-Russian Research Institute of Experimental Physics, Sarov, Nizhny Novgorod oblast, 607188 Russia

${ }^{h}$ Center for Theoretical and Experimental Particle Physics (CTEPP) of UNAB (Universidad Andres Bello), República 220 piso 2, Santiago, Chile

${ }^{i}$ KEK: High Energy Accelerator Research Organization, 1-1 Oho, Tsukuba, Ibaraki, 305-0801 Japan

${ }^{j}$ SOKENDAI: The Graduate University for Advanced Studies, 1-1 Oho, Tsukuba, Ibaraki, 305-0801 Japan

${ }^{k}$ Institute of Applied Problems of Physics, National Academy of Sciences of Armenia, Yerevan, 0014 Armenia

${ }^{l}$ Department of Molecular Physics, Faculty of Physics, Yerevan State University, Yerevan, 0025 Armenia

*e-mail: e.baldina@mail.ru

Received December 7, 2020; revised December 21, 2020; accepted December 21, 2020

\begin{abstract}
We present the scope of research of a new collaboration FLAP (Fundamental \& applied Linear Accelerator Physics collaboration) devoted to the study of the basics of electromagnetic interactions and new applications of controllable generation of electromagnetic radiation by relativistic electrons using functional materials.
\end{abstract}

DOI: $10.1134 / \mathrm{S} 1547477121030043$

\section{INTRODUCTION}

Since the first discovery of constituents of nuclear matter, the investigation of mechanisms of their interaction is the first priority task of physical science worldwide. Such great mega-science installations as the LHC (Large Hadron Collider, CERN), NICA (Nuclotron-based Ion Collider fAcility, JINR), etc., are built for these fundamental physics studies.

Another project at JINR is devoted to the creation of a unique electron accelerator LINAC-200 capable of producing electron beams with varying energy in a range from 26 to $200 \mathrm{MeV}$. It is planned to further increase the available electron energy up to $2 \mathrm{GeV}$. The high attractiveness of this facility to scientific groups both in Russia and other countries resulted in the establishment of a new scientific collaboration FLAP (Fundamental and applied Linear Accelerator Physics collaboration).

The FLAP collaboration is aimed at investigation of new fields of fundamental physics: generation of electromagnetic radiation by relativistic electrons. These tasks are important both for deeper understand- 
ing of the nature of electromagnetic interactions and development of advanced diagnostic tools for accelerated beams of charged particles, spintronic devices and novel superfast detectors of electromagnetic radiation. In particular, it is expected that the search of new light particles from the so called Dark sector (beyond the Standard Model) will be possible with the high intensity beam of the electron accelerator LINAC-200. Also, understanding of the origin of CP violation in terms of new axion-like bosons. Nondestructive diagnostics of circulating and extracted accelerated particle beams is one of the crucial issues in development of modern accelerators, including the NICA collider at JINR. Development and testing of such detectors, including those based on microchannel plates, will be addressed in the framework of the FLAP collaboration at LINAC-200. Also, neutron detectors for secondary neutron fluxes will be developed, tested and calibrated for applied research in the field of alternative power production concepts. Creation of pulsed neutron sources for investigation of extreme states of matter will also be addressed, as well as nondestructive neutron diagnostics for applied research in high pressure physics, study of metamaterials, and equations of state of matter. Problems of automation of accelerator complexes and physical experiments will receive good testing opportunities in this activity. LINAC-200 beams with well controllable properties may provide highly valuable information on radiation damage of DNA required for electron beam therapy of surface tumors. It should be underlined that the experimental studies planned in the framework of the collaboration have become feasible recently due to creation of new functional materials (metamaterials, topological insulators, Dirac semimetals, etc.), as well as new technologies (production of arrays of oriented nanoclusters with controllable parameters, nanoscale lithography, etc.), methods and detectors with high temporal, spatial and spectral resolution. All this allows one to expect fundamentally new results in this field.

The electron accelerator LINAC-200 is a unique facility with a number of controllable parameters: electron energy in a range from 26 to $200 \mathrm{MeV}$ (anticipated up to $2 \mathrm{GeV}$ ), macropulse duration from 30 to 3000 ns with a bunch duration of 1 ps, current from 0 to $60 \mathrm{~mA}$. The capability of adjusting the pulse repetition rate in a range of $2865 \pm 20 \mathrm{MHz}$. At present, this facility is the only one in the world with such flexible and reliably adjustable parameters. LINAC-200 is also an excellent facility for scientific education, which will definitely attract a large number of outer users, both from RF and other countries.

There exist several installations of a similar scale in leading international centers. The closest one is the scientific and educational complex CLEAR at CERN. The range of adjustable electron beam energies, however, is from 160 to $220 \mathrm{MeV}$ for a macropulse length from 10 to $200 \mathrm{~ns}$, which is lower by a factor of 15 than for LINAC-200.

The CLARA setup at Daresbury (Great Britain) with a fixed energy of up to $250 \mathrm{MeV}$ produces a single bunch with a duration of $300 \mathrm{fs}$ at a rate of $10 \mathrm{~Hz}$, which allows one to study certain aspects of electromagnetic radiation generation. This accelerator, however, is aimed at development of FEL laser technologies, and all experiments performed at this facility fit in the general strategy, which limits substantially the range of approached problems.

The LUCX facility at KEK (Japan) focuses on scientific and educational tasks. It is a linear electron accelerator which consists of a S-band 3.6 cell rf gun with a Cs2Te photocathode and linac booster. In cocalled fs-operation mode the series (1-4) of short (150 fs) $8 \mathrm{MeV}, 25 \mathrm{pC}$ micro bunches with variable $0-19$ ps separation and repetition rate of $3-12 \mathrm{~Hz}$ is generated [1]. In ps-operation mode up to 1000 bunches with an energy of $30 \mathrm{MeV}, 2.8 \mathrm{~ns}$ separation and 10ps duration are produced [2]. However, even with considered upgrade to $2.8 \mathrm{GHz}$ bunch repetition resulting in 10000 bunches in a macropulse it is impossible to achieve sub-ps bunch duration due to rf gun laser system limitations.

The demand for beam time at all the above mentioned facilities is extremely high, and the beam time schedule is filled for months ahead. This, of course, slows down research.

All this proves that LINAC-200 is capable of becoming a world leading facility, highly competitive in a number of fundamental and applied fields devoted to interaction of relativistic electron beams and electromagnetic radiation with matter.

\section{ADDRESSED SCIENTIFIC PROBLEMS}

The studies in the framework of the project FLAP are mainly devoted to extensively discussed lately terahertz radiation in a spectral range from 0.1 to $10 \mathrm{THz}$ because of a growing number of applications in this field, especially in medicine and security systems. Electromagnetic radiation of this spectral range is used in spectroscopy and nondestructive diagnostics, its applications permanently extending, since the varying chemical and biological systems possess selective absorption and reflection spectra. $\mathrm{THz}$ radiation is also applied for investigation of dynamics of nanomaterial formation, such as nanotubes and graphene. Low frequency radiation is used for detection of explosives and weapons, since nonconducting materials, including clothes, concrete, paper, are transparent for it. In this regard, generation of ultramonochromatic controllable radiation is highly preferable for increasing scanning precision and image contrast of scanned objects.

The project addresses promising fundamentally new directions of modern fundamental and applied science, 
especially new approaches and devices for generation of electromagnetic radiation based on functional materials. The following tasks are considered.

- Investigation of the processes of relaxation of electromagnetic response of materials based on topological insulators and Dirac semimetals with superhigh electron mobility (the electron mobility in these materials exceeds substantially that of graphene) under the action of bunched relativistic electron beam. The anticipated results will make the basis for development of contactless diagnostic tools for charged particle beams, radiators of electromagnetic radiation, and spintronic devices.

- Investigation of the possibility of controlling spectral and angular parameters of phonon response of metamaterials excited by bunched relativistic electron beams. The anticipated results will enable development of sources of electromagnetic radiation in the infrared and terahertz range controlled via variation of dielectric properties of the radiator, the target generating electromagnetic radiation under the action of a relativistic electron beam.

- Investigation of polarization radiation generation mechanism under the action of relativistic electron beam on surface structures with high local electron density, and study of the possibility of generation of electromagnetic radiation in charge and space coherence mode at electron beam interaction with such structures. The anticipated results will open new possibilities for development of sources of electromagnetic radiation based on targets-two-dimensional ordered structures from submicron clusters with high electron density which can be controlled via local charge density variation.

- Investigation of spectral and angular parameters of neutron yield at interaction of relativistic electron beam with matter, including ordered structures.

- Investigation of interaction of relativistic electron beams with various devices in the channeling regimes. The anticipated results will open new prospects in development of miniature devices for controlling spatial parameters of relativistic electron beams, which, in turn, will be a basis for development of deflectors, lenses, and undulators of a new generation.

- Investigation of the Dark Sector of the elementary particles in order to gain better understanding of the origin of the Universe.

- Investigation into novel methods of tumor therapy with electron and X-ray beams in the presence of photosensibilizing agents, such as porphyrins.

Experimental research in the framework of above tasks became feasible recently due to the creation of new materials (metamaterials, topological insulators, Dirac semimetals), as well as the development of new technologies (production of arrays of oriented nanoclusters with controllable parameters, nanoscale lithography), methods and devices for registration of radiation with high spatial and spectral resolution.
This gives grounds to expect fundamentally new firstclass results.

The modern status of research in this field worldwide is characterized by fragmentary studies along several directions included in the project. In particular, first observation of generation of reverse VavilovCherenkov radiation by relativistic electrons interacting with metamaterials was reported [3]. Note that reverse refraction was predicted long ago and was observed with photon beams. Unlike the above mentioned study, our project focuses on generation of radiation with the possibility of real-time control of spectral and angular distribution via variation of dielectric properties of the targets. The solution of this problem is of fundamental importance for future efficient sources of terahertz radiation.

Up to now, passage of charged particles through capillaries was studied for heavy positive particles (protons and ions) and low energy (to $10 \mathrm{keV}$ ) electrons. The existing models of electron interaction with material surfaces have a descriptive character. The novelty of the research in the framework of FLAP is as follows. First, these processes will be studied for electrons with energies $10-100 \mathrm{MeV}$ in the conditions of self-organized channeling, which addresses fundamentally different physical mechanisms of interaction. Second, the research will include electron passage through nano- and microcapillaries, including oriented arrays, which is extremely interesting from the point of view of elucidating the possibilities of formation of beams of relativistic electrons on a micron and submicron scale.

Generation of radiation by relativistic electron beam interacting with regions of high electron density, Dirac semimetals and topological insulators, is a fundamentally new field of research, both concerning the studied mechanisms and expected results, that would open ways for development of new approaches to controlled generation of electromagnetic radiation in a wide spectral range.

Six main directions are considered in the framework of the project.

The first direction addresses the processes of relaxation of electromagnetic response of materials based on topological insulators and Dirac semimetals (whose electron mobility exceeds substantially that of graphene) irradiated by bunched relativistic electron beams and development of contactless devices for diagnostics of charged particle beams, electromagnetic radiation, spintronic devices.

The key parameter in examination of electromagnetic interaction of charged particles with condensed medium is the mobility of the medium electrons, which is directly related with the rate of generation of electromagnetic response of the medium on the perturbation. It should be noted that a noticeable progress has been achieved in the development of multifunctional materials, including materials with superhigh 
charge mobility. The analysis of modern achievements in this field showed that one of the promising areas is the group of semiconductor materials $\mathrm{A} 2 \mathrm{~B} 5$, due to the discovery of the properties of 3D Dirac semimetal in Cd3As2 monocrystals.

Cd3As2 monocrystals are 3D analogues of such well known 2D material as graphene. Superhigh mobility of charge carriers, $9 \times 10^{6} \mathrm{~cm}^{2} \mathrm{~V}^{-1} \mathrm{~s}^{-1}$ at $5 \mathrm{~K}$, is observed in Cd3As2 monocrystals. The lifetime of charge carriers is longer by a factor of $10^{4}$ than the quantum lifetime [4]. Fermi velocities of 3D Dirac fermions in Cd3As2 are higher by about a factor of 1.5 than those in graphene. Experiments with angular resolution photoemission spectroscopy (ARPES) proved the presence of a surface electron structure typical for a spatial (3D) Dirac semimetal. Practical application of Cd3As 2 has been addressed. The results of testing the metal-Cd3As2-photodetector metal prototype demonstrating a sensitivity of $5.9 \mathrm{~mA} / \mathrm{W}$ with a response time of about 6.9 ps without any special device optimization were reported in 2017. The main element-receiver of electromagnetic radiation-was the substrate with Cd3As2 nanoplates or nanowires. Already at the prototype stage the response in a wide band from 532 to $10.6 \mu \mathrm{m}$ was achieved with a range of potential detection extended to far infrared and terahertz wavelength [5]. The combination of the possibility to vary the width of the forbidden band and the band structure from reverse, via gapless state to positive forbidden band together with topological properties, Dirac cones, and massless fermions with an exiton Boron radius of $\sim 47 \mathrm{~nm}$ makes the study of the properties of such materials extremely interesting, and the application of such materials promising.

The above properties of Cd3As 2 look quite promising for investigation of photon response of a material on perturbation introduced by a bunched beam of relativistic particles. This field of research is promising for applications aimed at development of sources and sensors of electromagnetic radiation, spintronic devices. Superfast response and high sensitivity of materials of Cd3As2 family is a unique feature for creation of contactless devices for diagnostics of charged particle beams, which is one of the main problems of modern accelerator physics.

The second direction focuses on the problems of controlling spectral and angular characteristics of photon response for metamaterials irradiated by bunched relativistic electron beams, as well as the development of unique highly monochromatic and narrowly directed controllable sources of electromagnetic radiation of the new generation.

Modern methods of electron lithography, deposition, and etching ensure the creation of micro- and nano-structures with sub-nanometer size. Such structures may possess unique optical properties determined by the shape, size, and order. If the size of a structure is comparable with the wavelength of elec- tromagnetic field or interacting electromagnetic radiation, such substances are called photon crystals, and if the size of a structure is much smaller than the wavelength, they are called metamaterials. Such nanostructures as ring resonators can be excited by incident radiation or a relativistic particle passing by, and affect incident radiation in an unusual way, for example, cause reverse refraction or focusing violating the diffraction limit. Since the size of a structured cell is much smaller than the wavelength, the field feels such medium as a solid with negative effective refractive index. The flexibly chosen parameters and special electromagnetic properties attracted attention of experts in the accelerator field. Metamaterials are examined as absorbers of high harmonics in microwave resonators, as components in new structures for field amplification or attenuation, as structural targets for generation of electromagnetic radiation and control of its parameters. Generators based on metamaterials may increase radiation generation efficiency in $\mathrm{THz}$ wavelength range.

This project addresses the study of the possibility of creation of targets for relativistic electron beams capable of efficient generation of electromagnetic radiation with on-line controllable spectral and angular characteristics. This feature has been predicted theoretically but not implemented experimentally yet. The area of application of such sources is vast.

The third direction is devoted to the investigation of polarization mechanism of electromagnetic radiation in surface structures with high local electron density irradiated by relativistic electron beams and the possibility of generation of radiation in the charge-space coherence mode.

This direction is focused on a fundamentally new approach to generation of electromagnetic radiation based on interaction of an accelerated electron beam with spatially ordered regions of high electron density. The mechanism is based on the polarization phenomenon when the medium perturbed by the Coulomb field of the accelerated particle, the relativistic electron in our case, emits radiation. The closest research is connected with the study of Smith-Purcell radiation generated when a bunched electron beam moves above diffraction gratings of different structure, which is fundamentally different from the problem considered in this project both with respect to the physical processes and technical implementation of experiment. The most important features making the research in the framework of FLAP unique are as follows. An active controllable medium representing periodic regions of high electron density is considered as the radiator (target); the charge density of free electrons in these regions is rather high, and they create a field of order of $100 \mathrm{MeV} / \mathrm{cm}$. Unlike the standard case of Smith-Purcell radiation, the target (diffraction grating) was made from a homogeneous conductor (targets were grounded in experiments). In order to 
obtain classical high intensity short-wavelength Smith-Purcell radiation, a bunched electron beam with a bunch length shorter than the wavelength of the considered radiation is required (the intensity of generated radiation increases quadratically with increasing number of electrons in the bunch), which is a substantial technical difficulty for radiation with a frequency above $1 \mathrm{THz}$. Of course, Smith-Purcell radiation may be generated by a single electron interacting with a target, but the yield of such radiation is negligible, and for the hard spectral region of interest radiation intensity increases linearly with respect to electron beam current. Unlike the classical case, in the approach considered here, coherent radiation can be generated upon excitation of regions of high electron density by one passing electron, and the yield of this radiation will be much higher than that in the classical mode of Smith-Purcell radiation. It should be noted that if a bunched electron beam were used in the considered approach, radiation intensity would further increase.

An important technological achievement in development of targets with regions of high electron density is the possibility to control electron density of separate regions, which opens new capabilities of controlling the parameters of the surface structure in such targets, for example, based on selective activation of a set of selected regions for creation of the required structure. This feature, in turn, opens new capacities in controlling spectral and angular characteristics of generated radiation.

Thus, the proposed study carries fundamental scientific novelty in the field of the physics of radiation produced by charged particles, namely, polarization radiation at interaction of accelerated particles with ordered structures of high electron density. The novelty in the applied field consists in development of the basis for fundamentally new laboratory-scale controllable high intensity sources of electromagnetic radiation.

The fourth direction is aimed at investigation of interaction of relativistic electron beams with nanocapillary and corrugated devices, in the electron channeling mode.

Interaction of charged particle beams with dielectrics was mainly examined from the point of view of defect and damage formation. For example, if charged particles hit a dielectric surface, the surface becomes charged; charging up of separate parts of spacecrafts may result in electrical breakdown and damage of dielectric elements and onboard equipment. In electron microscopy normal incidence of electrons on a non-conducting surface impairs the analysis of this surface, sometimes making it completely impossible because of its overcharge. In the case of grazing (close to zero angle) beam incidence, however, the surface may form a charged layer capable of preventing the interaction of the main part of the beam with the surface. The studies aimed at efficient control and focus- ing of positively and negatively charged ions and electrons using nano- and microcapillaries have been under way since the beginning of 2000se. One of the significant results was the ability to increase the current density of helium ion beam at the output of the tapered glass channel by four orders of magnitude [6]. Such ion focusing is used at present in biological studies and analysis of solid structures. Similar results for an electron beam were reported in [7]. Interaction of grazing electrons with dielectric surfaces, however, differs substantially from the interaction of ion beams with surfaces.

The extensively study of electron beam interaction with dielectric surfaces based on comprehensive description of the processes involved in this interaction has been under way worldwide. The main difficulty in description of grazing interaction of electrons with matter is the surface (self- and induced) conductivity. The value of this conductivity depends on different factors, such as electron incidence angle, energy and current, type of dielectric. Measurement and variation of these properties is a separate experimental task.

Thus, in spite of a rather high potential in the field of applied research, these experimental studies lack reproducibility and progress in development of theoretical models of the considered processes, which imposes certain constraints on development of technical devices.

This project will address the processes of formation of a self-consistent electric field on inner walls of nano- and micro-capillaries under the action of relativistic electron beam passing through them. The processes of passage of such beams through corrugated axially symmetric structures and arrays of oriented capillaries will also be studied. Of special interest are the conditions of stable particle channeling in such targets. The main applications of anticipated results are connected with development of new approaches to controlling relativistic electron beams, in particular, formation of periodic trajectory and focusing.

The fifth direction covers several applied problems. They are the development and testing of advanced detectors for nondestructive beam diagnostics, as well as development and calibration of novel detectors of secondary neutron fluxes. Development of modern principles of controlling large-scale physics facilities, involving machine learning. Creation of sources of secondary particles, such as neutrons, with controllable properties for studies in the field of high pressure physics and equations of state. Investigation and development of modern methods and approaches in radiation medicine with controllable electron and gamma beams.

The sixth direction focuses on studies of sensitivity to new physics beyond the Standard model. In particular, it is planning to look for light particles: axionlike, dark-photon-like and Higgs-boson-like parties in 
$\mathrm{MeV}$ range. One of the main differences with similar ongoing projects in US and Germany is the use of sick heavy targets.

\section{EXPERIMENTAL AND THEORETICAL BACKGROUND}

The research discussed here is based on the unique expertise of the project team in the field of interaction of relativistic charged particles with matter and external electromagnetic fields. The formulated tasks are well grounded by well known fundamentals of electrodynamics and physics of condensed state. Some planned experiments are based on previous experimental research of the team members, as well as theoretical simulations. The experimental basis of the project consists in the experience of the team members acquired at different accelerators (LINAC-200 at JINR, CLEAR at CERN, PETRA at DESY, CLARA at Daresbury, LUCX at KEK, "Pakhra" at Lebedev Physical Institute) in measurements in a spectral range from several $\mathrm{GHz}$ to gamma radiation.

The basic scientific methods and approaches are as follows.

The source of accelerated electrons is the linear electron accelerator LINAC-200 with an electron energy from 26 to $200 \mathrm{MeV}$. The accelerator is equipped sufficiently to serve as the basic experimental installation of the project.

High sensitivity detectors based on such units as Schottky diodes, pyroelectric sensors and bolometers will measure spectral and angular characteristics of radiation in a range from $1 \mathrm{GHz}$ to $10 \mathrm{THz}$. High spectral resolution $\left(\sim 10^{-7}\right)$ in a range up to $50 \mathrm{GHz}$ will be achieved using a spectrum analyzer with pyramidal antenna, and in a range to $600 \mathrm{GHz}$ by a heterodyne system.

Photoelectron multiplier detectors (in air), as well as secondary electron multipliers and microchannel plates (in vacuum) will measure spectral and angular characteristics of radiation in a range from infrared to ultraviolet. Sensitive CCD or CMOS cameras with high spatial resolution will be used in the optical range.

Two registration systems will be used to measure spectral and angular characteristics of radiation in a range from vacuum ultraviolet $(110 \mathrm{~nm})$ to soft $\mathrm{X}$-rays $(1 \mathrm{~nm})$ :

- grazing incidence spectrometer McPherson 248/310G and electron multiplier Hamamatsu R5150-10, the combination of which devices is capable of measuring spectra with an energy resolution of $0.1 \%$;

- vacuum-compatible semiconductor spectrometers Amptek with thin windows X123SDDFASTC1 and X123SDDFASTC2 are capable of measurements in the required spectral region with a counting rate of up to $10^{6}$ photons/s and an energy resolution of about $100 \mathrm{eV}$.
Vacuum-compatible semiconductor spectrometers AmptekX123SDDFAST (spectral range 1-20 keV, counting rate up to $10^{6}$ photons/s, energy resolution $125 \mathrm{eV}$ for Fe55) and AmptekX123CdTe (spectral range $5-120 \mathrm{keV}$, counting rate $10^{5}$ photons/s, energy resolution about $850 \mathrm{eV}$ for an energy of $122 \mathrm{keV}$ ) will be used for measurement of spectral and angular characteristics of $X$ rays $(1-100 \mathrm{keV})$.

Scintillation spectrometer SDMF1206 with neutron contribution discrimination based on analysis of the pulse shape will be used to measure gamma spectrum $(0.1-6 \mathrm{MeV})$; this will also provide neutron background monitoring.

Semiconductor spectrometers Ortec equipped with appropriate spectrometric NIM electronics will be used for registration of charged particles via their ionization losses.

Background suppression presents the main difficulty in modern experiments. The following background components should be taken into account: parasitic electromagnetic fields, charged component (scattered and secondary charged particles), neutral component (X-rays, gammas, neutrons). The following methods will be used in background suppression: screening of the measurement equipment and a separate grounding for it; strong collimation of the observed signal to cut off the background of particles emitted by elements of the experimental setup; synchronization of data accumulation and time structure of electron beam in order to cut off the background during idle time; installation of magnetic deflectors for the charged background component. All these methods have been successfully applied by the project team members at different experimental setups, including those incorporated in the project.

Most experiments require vacuum measurements which means that the positions of detectors and target, as well as other elements of the experimental setup in vacuum should be controlled remotely and in vacuum. This can be ensured by using vacuum moving holders with step motors Phytron VSS42 (manufactured by Standa, models 8MR190V-2, 8MR191V-2, 8MT175V-150), as well as application of vacuum-compatible detectors (to $10^{-6}$ Torr) manufactured by Amptek, units Vac Option A2 X-123 FAST SDD $\left(25 \mathrm{~mm}^{2} / 500 \mathrm{um} / \mathrm{C} 1\right.$ window); Option 6 Configuration, PA210 preamp with housing, 9DVF vacuum feedthrough connector on a 2.75" SS conflat, ACH-417 (4" L shielded cable) and ACH-411 (12" L shielded cable)).

All experiments will be preceded by theoretical calculations and mathematical modeling of expected radiation parameters in order to determine the optimal experimental conditions. Analytical calculations will be based on classical approaches, first of all using Maxwell equations with corresponding boundary conditions determined by the experiment geometry and the conditions of interaction of relativistic electrons with targets. Polarization mechanisms of radiation 


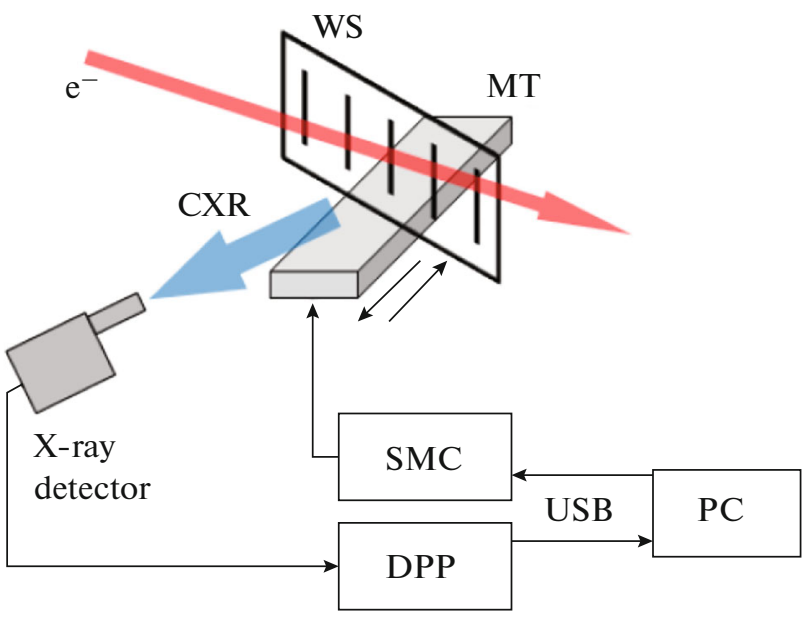

Fig. 1. Schematic diagram of experiment [1]. WS-frame with parallel wires from different materials $(\mathrm{Ti}, \mathrm{Cu}, \mathrm{Mo}$, $\mathrm{W}, \mathrm{Pt}) ; \mathrm{MT}-$ motorized linear platform moving the wired frame across the beam axis; CXR-characteristic X-rays produced at interaction of electron $\left(\mathrm{e}^{-}\right)$beam with the wires; SMC, DPP, USB, and PC-signal readout and processing devices.

generation will be described with the help of the theory of polarization bremsstrahlung radiation of relativistic charged particles. Mathematical simulation will apply such well known and physically reliable software as GEANT and CST-Studio.

\section{PROJECT TASKS}

\section{Task 1}

Testing of the method of measurement of the position and shape of charge density distribution for relativistic electron beams using a monitor based on measurement of spectra of characteristic X-rays. Characteristic $\mathrm{X}$-rays are manifested in the form of quasimonochromatic $X$ ray lines whose position is determined by atomic structure. This specific feature has been widely used, for example, in X-ray fluorescence analysis for determination of elemental composition of matter. An approach to measurement of the beam position and cross section shape based on a special thin wire target consisting of wires from materials with different elemental composition. X-ray signal will be registered by an energy dispersive spectrometer discriminating characteristic lines of generated radiation, thus defining the sought characteristics of electron beam. A modification of the proposed study was implemented in [8]. Figure 1 shows the schematic diagram of this experiment.

\section{Task 2}

Study of transversal of relativistic electrons through micro- and nano-capillaries, including structured ones. Investigation of the process of passage of accelerated charged particles through a system of narrow dielectric channels has become a promising research topic in the last decade. This problem has mainly been studied for ion beams, while for electron beams the research has just started. The basis of the considered processes for ions is the formation of self-consistent charge distribution on inner capillary walls due to the interaction of charged particles with these capillary structures. The mechanism of the processes of interaction of a beam of relativistic electrons with the inner walls of dielectric channels requires study.

At present, the only result obtained by the joint team of Belgorod State University and KEK demonstrated the possibility of focusing a $8 \mathrm{MeV}$ electron beam traversing a corrugated capillary [9]. An electron energy of $8 \mathrm{MeV}$ is the record one in this field of research. It is interesting to continue the studies with higher energy electron beams because the effective impact parameter of the beam interaction with inner walls grows with increasing electron energy. Figure 2 shows the schematic diagram of the experiment and the basic results, namely, the discovered correlations of the capillary axis position with respect to the incident beam axis and the position of maximum of the passed beam (black points) and the horizontal beam focusing (red points). The beam size at the input of the capillary was $0.8 \mathrm{~mm}$. It can be seen from Fig. 2 that when the channel axis shifts with respect to the beam axis (their position coincides at zero), the maximum of the traversed beam also shifts; the plot also clearly indicates the focusing effect of the dielectric channel.

\section{Task 3}

Study of diffraction mechanisms of radiation in vacuum ultraviolet and soft $\mathrm{X}$-rays in the conditions of anomalous dispersion of diffracted photons. The addressed problems have a fundamental character, they are aimed at investigation of diffraction methods of emission by relativistic charged particles determined by coherent scattering of fields of real and virtual photons on atomic and layered structures in the conditions of anomalous matter dispersion. Anomalous dispersion means the energy region of observed photons for which the real part of dielectric permeability is larger than unity, which, in particular, opens the channel of particle energy loss via Cherenkov radiation (usually, the real part of dielectric permeability is smaller than unity in the spectral region from vacuum ultraviolet and harder). This specific feature is mainly observed in a narrow spectral range near the matter photoabsorption edges, which provides generation of quasimonochromatic radiation.

The interest to this field is determined, first of all, by verification of existing theories describing the mechanisms of diffracted bremsstrahlung radiation, diffracted transition radiation, parametric $\mathrm{X}$-rays, and Cherenkov radiation emitted at the same time [10]. In 
(a)

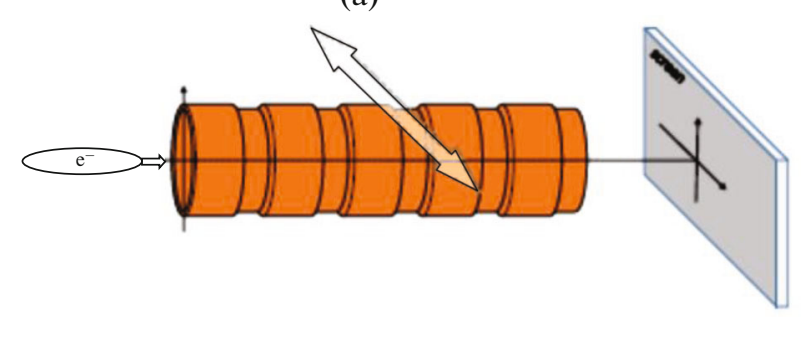

(b)

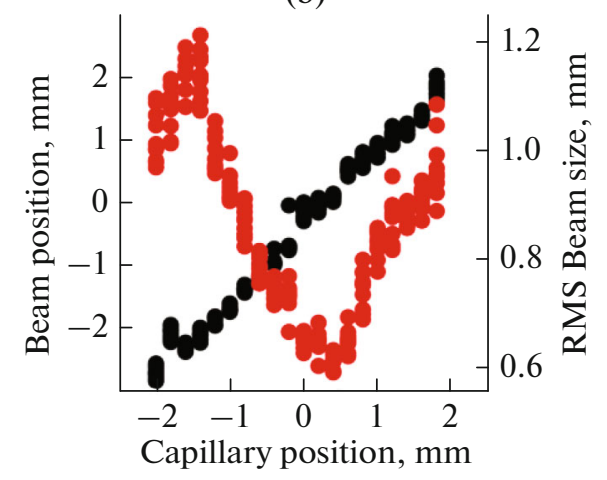

Fig. 2. (a) Schematic diagram of experiment. (b) Experimental results: (black points) capillary axis position versus incident beam axis and (red points) horizontal beam focusing [9].

(a)

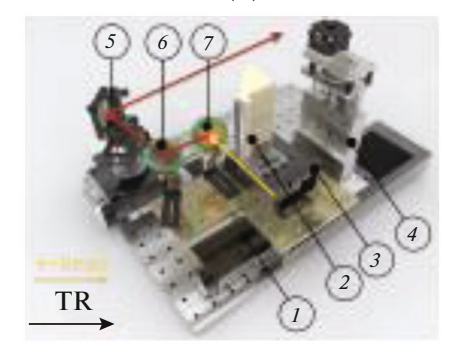

(b)

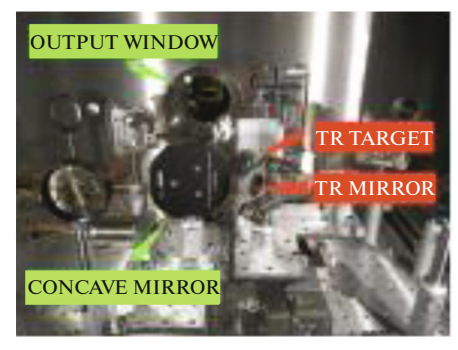

(c)

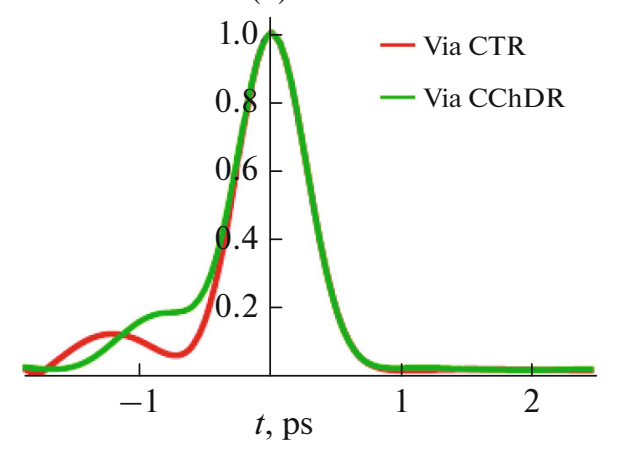

Fig. 3. (a) Setup inside vacuum chamber: (1) horizontal positioning stage, (2) ChDR target (method with non-invasive coherent Cherenkov diffraction radiation described in ref. above, (3) tip-tilt stage, (4) vertical positioning stage, (5) concave mirror, (6) TR steer mirror, TR radiator; (b) photo of experimental setup inside a vacuum chamber; (c) longitudinal charge distribution measured via coherent transition (CTR) and coherent Cherenkov (ChDR) radiation.

particular, in the considered case the existing theories predict anomalously large yield of parametric X-rays.

\section{Task 4}

Measurement of average micro-bunch length and bunch train length. The length of a separate bunch and the number of bunches in a train play a key role in a number of applications of charged particle accelerators, including for generation of high intensity ultramonochromatic radiation in the terahertz spectral range.

The methods based on measurement of spectra of coherent radiation at the radiation wavelength comparable or larger than the particle bunch length are the most promising ones, since they have no fundamental limitation on their resolution. It is proposed to use Vavilov-Cherenkov radiation in the diffraction regime, when a fast charged particle passes near a dielectric [11] as the generation mechanism which possesses a number of advantages, as compared to other mechanisms, namely, narrow angle, intensity proportional to the radiator length, non-invasive character of generation. This method was tested at the KLARA setup (Daresbury, Great Britain) [12].

Figure 3 illustrates the experimental setup and results.

The solution of this task will result in the creation of longitudinal diagnostics for the linear electron accelerator LINAC-200, will provide necessary information for solution to tasks 5 and 6 (see below), and will prove the efficiency of the method of longitudinal diagnostics using coherent Vavilov-Cherenkov radiation.

\section{Task 5}

Generation of high intensity ultramonochromatic radiation in $\mathrm{THz}$ ad sub- $\mathrm{THz}$ spectral range in the "super-radiation" regime. Generation of high intensity monochromatic radiation in the $\mathrm{THz}$ and sub- $\mathrm{THz}$ range with adjustable frequency is a topical task due to an increasing number of research fields in chemistry and biology. Existing sources have their disadvantages 
(b)

Interferometer mirror displacement, $\mathrm{mm}$

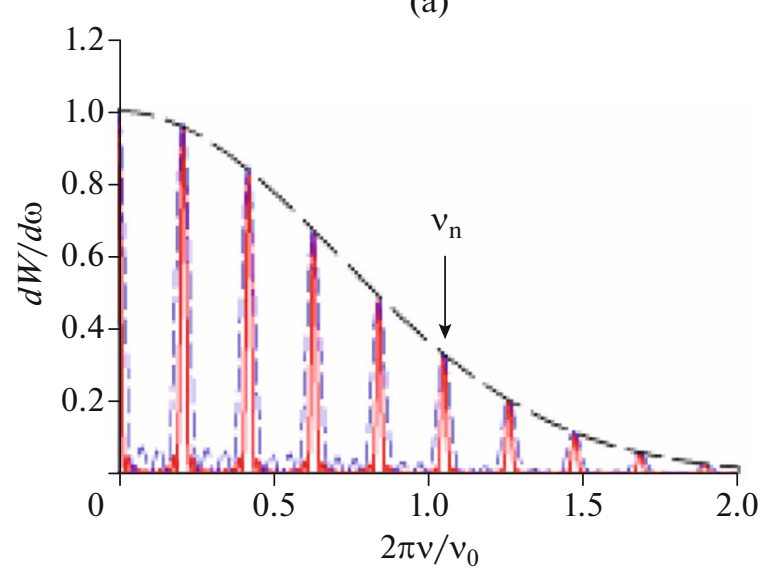

Fig. 4. (a) (Black dashed line) Normalized CTR spectrum from a single bunch, (red solid line) CTR from a train for $N_{\mathrm{b}}=10$, and (bold blue line normalized to the line for $N_{\mathrm{b}}=10$ ) for $N_{\mathrm{b}}=4$. (b) Spectrum reconstructed from the measured CDR interferogram shown in insertion.

(a)

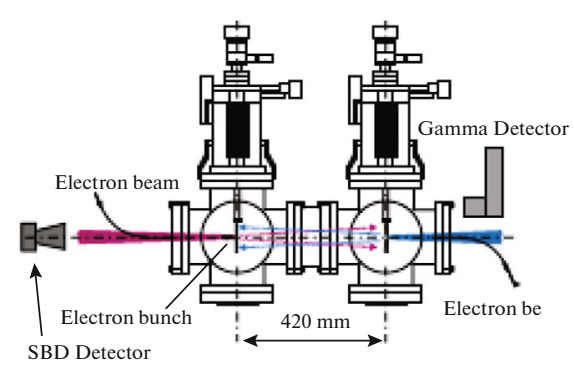

(b)

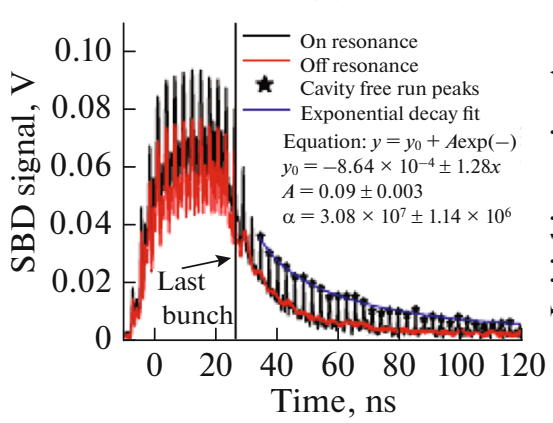

(c)

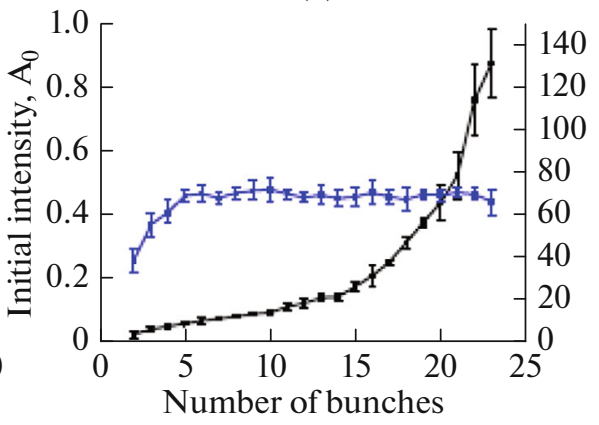

Fig. 5. (a) Experimental layout. (b) Radiation stored in the resonator. (c) Intensity as a function of the number of bunches in the train.

which should be resolved in an integrated manner to achieve high intensity, frequency above $1 \mathrm{THz}$, ultrahigh monochromaticity, and possibility of frequency adjustment.

At present, the most promising sources of $\mathrm{THz}$ radiation are based on accelerators. It is necessary, however, to optimize the generation regime. We propose to use coherent diffraction Vavilov-Cherenkov radiation in the super-radiation regime. Coherent radiation is generated at the wavelength comparable or larger than the electron bunch length. In this case, intensity is proportional to the squared total charge in the bunch. The super-radiation regime occurs if the electron beam is a train of a large number of equidistant bunches.

This regime was observed at the microtron (6.2 MeV) of the Tomsk Polytechnic University (see Fig. 4) [13, 14] using coherent transition radiation. A long bunch and low electron energy, however, prevented from reaching $\mathrm{THz}$ frequencies.
It is proposed to demonstrate generation of narrow lines of sub-THz and $\mathrm{THz}$ radiation at the LINAC-200 accelerator. Such source is attractive to users in other fields for unique studies connected with interaction of $\mathrm{THz}$ radiation with matter.

\section{Task 6}

Stimulation of induced radiation at generation of THz radiation. This is an exploratory research. The efficiency of sources of electromagnetic radiation based on linear accelerators is very low. Stimulation of induced radiation would increase radiation yield. Some energy (from first bunches) would circulate inside a radiator, stimulating loss from the following bunches.

A similar scheme was implemented using the mechanism of induced diffraction radiation (see Fig. 5) [15]. We propose to use this mechanism at LINAC-200. 


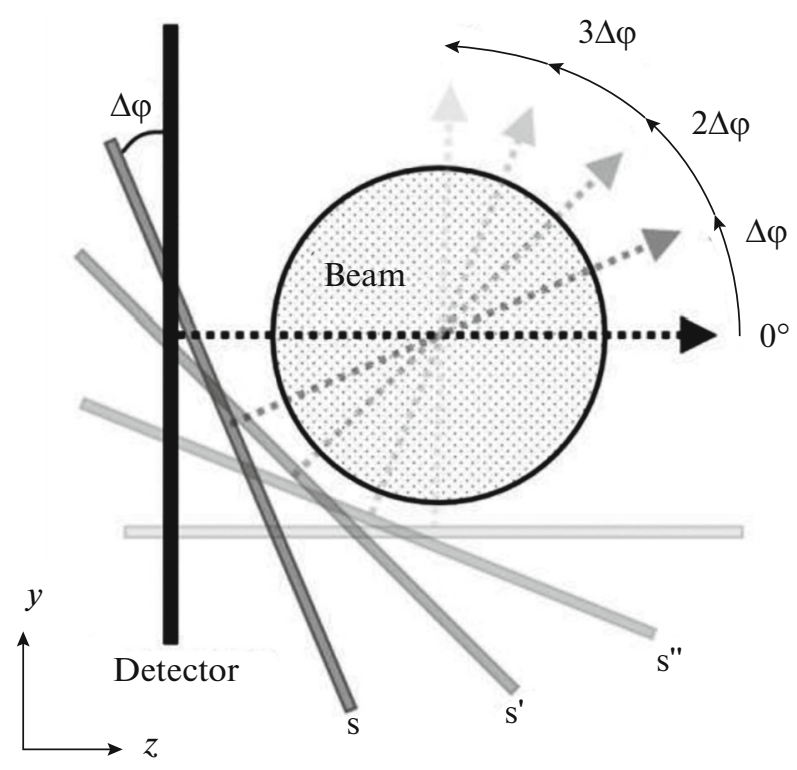

Fig. 6. Scheme for measurement of electron flux density in the plane perpendicular to the beam based on multi-angle scanning.

\section{Task 7}

Proof-of-principle experiment for measurement of transverse electron beam profile based on multi-angle scanning. Transverse beam profile is an important parameter defining the main beam properties, and therefore, the processes of its interaction with matter. Development of methods for determination of this parameter is an important task in high energy physics.

A method for measurement of the beam profile is based on reconstruction of data obtained in multiangle scanning [16]. Figure 6 shows the scheme for measurement of particle flux density in the beam which is based on multi-angle scanning.

The beam profile in the perpendicular plane is determined via reconstruction of the radiation flux intensity as a function of coordinates in the measurement plane and the intensity of radiation hitting the detector as a function of its position.

\section{Task 8}

Measurement of the spatial shape of electron beam using multi-angle scanning. The proposed approach to measure the spatial structure of electron beam is based on the method described in Task 7 [16]. The difference is that intensities in particular positions of a detecting element are measured in a time-resolved way, as the bunch traverses the detector. Depending on the beam properties and detecting equipment, this approach may provide measurement of the shape of a separate bunch (see Fig. 7a) or a train (Fig. 7b).

If the signal time evolution is measured, the analysis of the collected data will provide reconstruction of the beam cross section in each time interval. A set of such reconstructions will give the beam shape.

\section{Task 9}

Applied studies on testing promising detector types. Taking into account the capability of variation of the time structure of the electron beam from units of $\mathrm{Hz}$ to $\mathrm{MHz}$ and a short pulse duration of up to $50 \mathrm{~ns}$, it is proposed to study loading characteristics (recovery time) of detectors based on chevron (dual) assemblies of microchannel plates (MCP). Such detectors have been extensively used at LNP and LHEP JINR for nondestructive monitoring of circulating and extracted accelerated particle beams [17, 18]. Such detectors will also be applied at the constructed Booster, Nuclotron, and storage rings of the NICA acceleration complex. A small-angle scattering detector based on MCP for the experiment SPD is under development [19]. It is proposed to perform a series of measurements of pulsed (up to 10000 particles in $50 \mathrm{~ns}$ ) loading of detectors depending on the pulse repetition rate. This study is performed in close cooperation with LLC "Baspik", manufacturer of MCP.

It is proposed to test some promising neutron detectors at secondary neutron fluxes produced in interaction of relativistic electron beam with target nuclei, in the pulsed irradiation mode. Simultaneous registration of time-of-flight neutron spectra and with the tested threshold neutron detectors will provide (a)

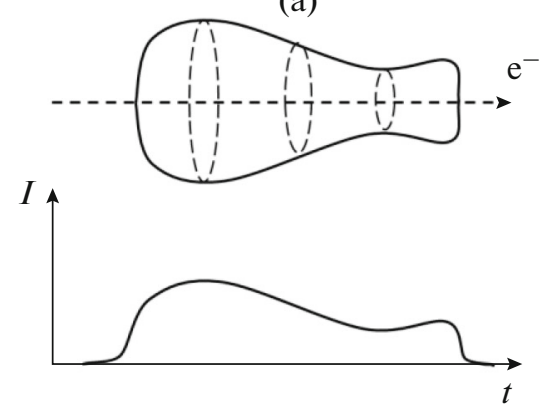

(b)
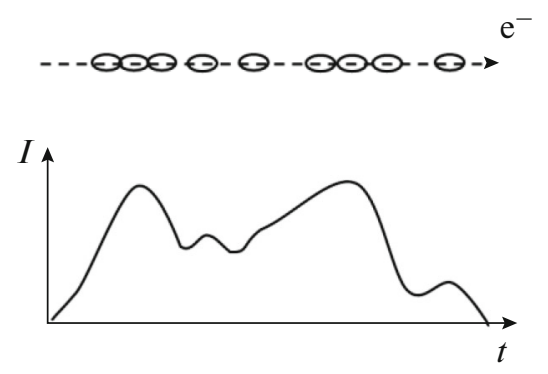

Fig. 7. Schematic diagram of data acquisition for an electron beam traversing a scanning element. 
testing and calibration of new types of spectrometric neutron detectors, in particular, for experiments of "Energy+Transmutation" collaboration [20].

It is also proposed to continue studies of ALPIDOtype micropixel detectors at LINAC-200 beams and perform exploration experiments with thin targets of about $1 \mu \mathrm{m}$ for precision measurement of angular and momentum dependences of positron production by relativistic electron beams.

\section{Task 10}

Implementation of modern approaches to automation of physical experiment, including low-level computer monitoring of the acceleration complex and all experimental stations, involving graphic user interface and machine learning. The quality of experimental data directly depends on the possibility to control and monitor charged particle beam parameters. All standard diagnostic methods should be reliable, and results should be reproducible, which is strongly connected with the control of parameters of the accelerator complex and the option of variation of one or several parameters in a controllable way. All this is also true for experiments on generation of radiations and development of new diagnostic methods.

Usually, accelerator control systems have several levels of organization and represent distributed (decentralized) networks with data and/or signal exchange between them. The lowest level is the safety interlock system. This level is implemented according to an organization rules, it is a feedback system based on microcontrollers. The intermediate level includes the time synchronization level, the microwave system, and the data acquisition and digitalization system. This level is constructed using multichannel electronics (the most widely spread systems are NIM, VME, CAMAC, PXI, PCI), or field programmable gate arrays (FPGA) and contains program components determined by a particular installation and research program. The upper level usually represents a graphic interface used to operator - control and monitor the accelerator parameters. This level also includes data processing scripts (averaging, normalization, FFT, etc.), data plotting and real-time data storage. Machine learning can also be implemented at this level, which is, in essence, multiparametric optimization of one or several parameters via adjustment of other parameters of the accelerator complex.

Since such a system consists of a rather large set of spatially distributed controllers, computers, and sensors, a special data communication network protocol EPICS was developed at ANL. At present, EPICS is used at $90 \%$ of accelerator centers. The system TANGO used in many European centers is the direct competitor of EPICS, however, its technical support is much less advanced.
The KEK team participating in the project possesses experience in all the above, except for machine learning, which has just come into focus. The groundwork in this field is based on such reliable free software as Python, Qt, PyQt, which are independent of a system platform (Linux, Windows, or MacOS) and are readily scalable.

We propose joint development/upgrade of the LINAC-200 control system. It is anticipated that these efforts will have a noticeable effect on the stability of accelerated beam parameters, their reproducibility, and will accelerate putting into operation experimental stations.

\section{Task 11}

Study of a possibility of constructing a compact pulsed neutron source based on an accelerator and a light target for experiments in the field of physics of extreme states of matter. The following problems will be addressed:

- Measurement of temperatures of shock compressed metals using neutron resonance spectroscopy.

- Formation of hydrodynamic Richtmyer-Meshkov instabilities in shock-compressed metals.

- Dynamic neutronography of shock-compressed metals.

- Study of crystalline structure of shock-compressed metals via neutron scattering on crystalline lattice.

- Neutron radiography and introscopy.

Nondestructive neutron diagnostic methods are required in fundamental and applied research in the field of high pressure physics, for obtaining and study of new metamaterials with unique properties, study of atomic structure on a nanometer scale, and more precise determination of equations of state of matter.

It is proposed to obtain a pulsed neutron flux via spallation reaction when the beam interacts with beryllium or lithium target with low photoneutron reaction threshold. Neutrons will be moderated to thermal spectrum using a moderator (Fig. 8).

Neutron flux through an object will be recorded using two methods: by fast time of flight pulsed neutron detector (Fig. 9) for investigation of neutron spectra, and by neutron-sensitive camera based on scintillation converters with cadmium and gadolinium salts combined with a high speed camera [21] (Fig. 10) for obtaining neutron radiography images. Twodimensional gamma and $\mathrm{X}$-ray fields will be detected using a nanosecond gamma camera based on direct conversion of radiation in MCP [22] (Fig. 11).

\section{Task 12}

Search for new light particles at LINAC-200. The high intensity electron beam of LINAC-200 can be 


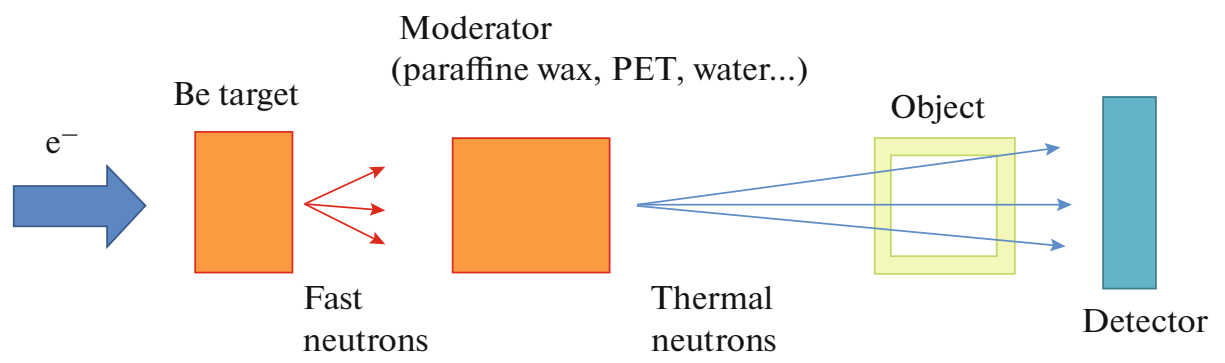

Fig. 8. Schematic diagram of neutron flux generation and detection.

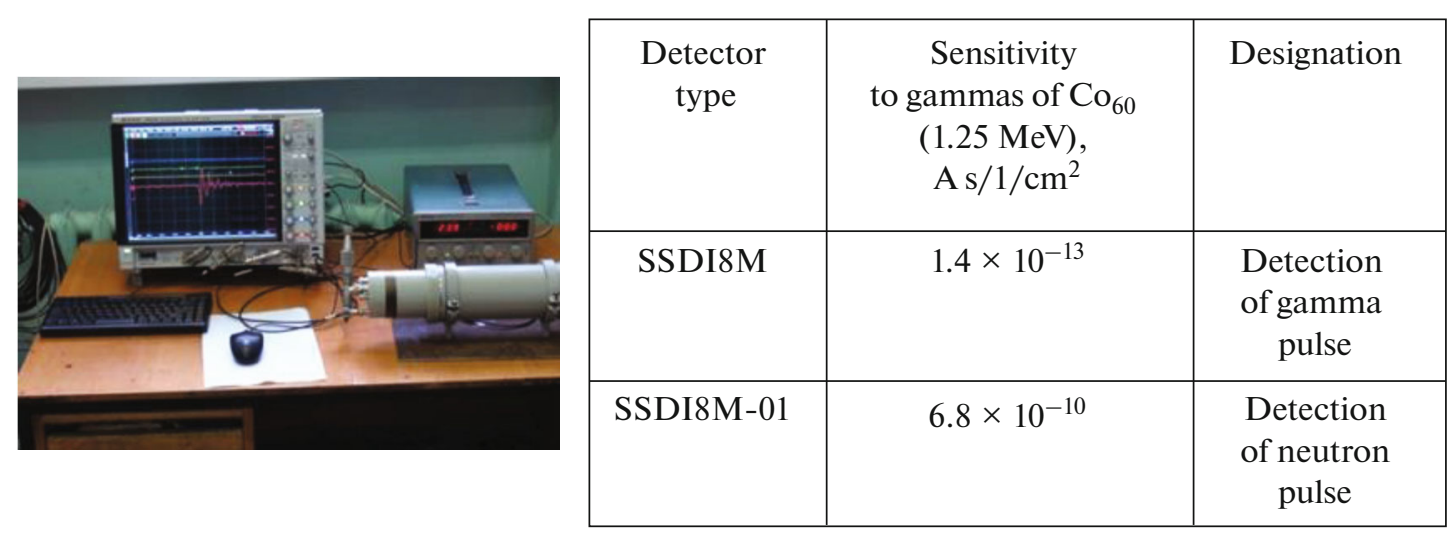

Fig. 9. Gamma-neutron detectors.

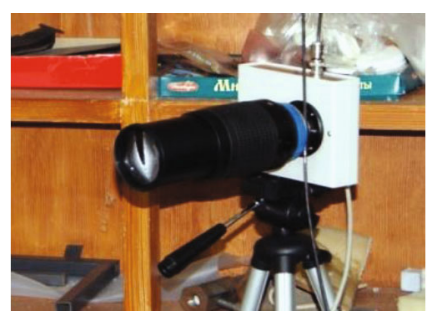

- 8 shots

- 96 ns interval between shots

- 96 ns exposure time

- $64 \times 128$ resolution

Fig. 10. High speed camera.

used for the search of new light particles beyond the Standard Model (SM) of elementary particles in a mass range of 1-20 MeV. The so-called Dark sector of elementary particles has various candidates (dark pho- ton, neutralino, etc.) for explanation of Dark Matter origin of our Universe (see for a review, e.g., [23]). If there are forces beyond gravity and the SM particles between Dark Matter and SM particles, then one can

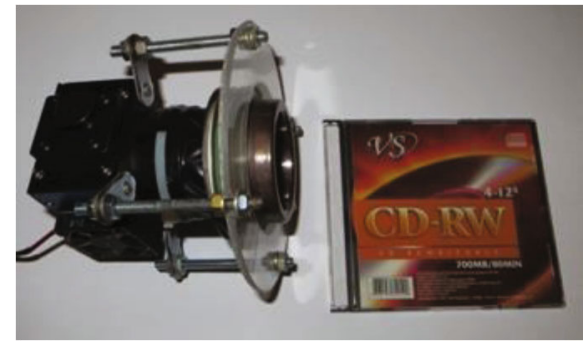

- Spectral range: $0.01-300 \mathrm{keV}$

- Working area: $\varnothing 4 \mathrm{~cm}$

- Scanning depth: $2 \mathrm{~cm}$ steel

- Dynamic range: 12 bit

- Resolution: $200 \mu \mathrm{m}$, contrast $30 \%$

- Exposure: $5 \mathrm{~ns}$

Fig. 11. Nanosecond gamma camera based on MCP. 
(a)

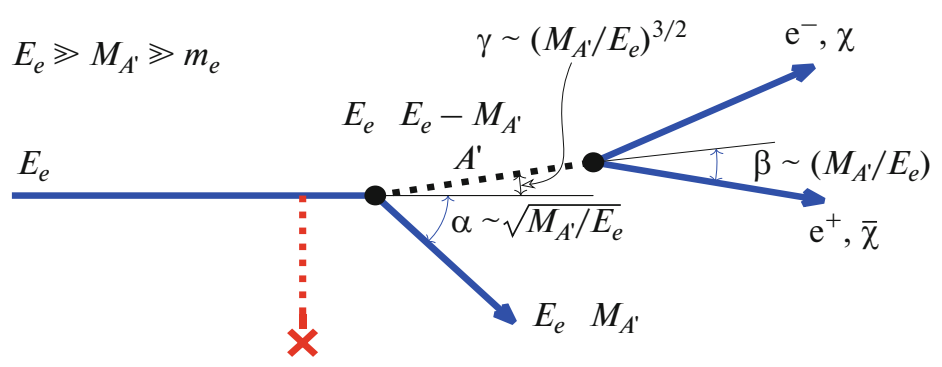

(b)

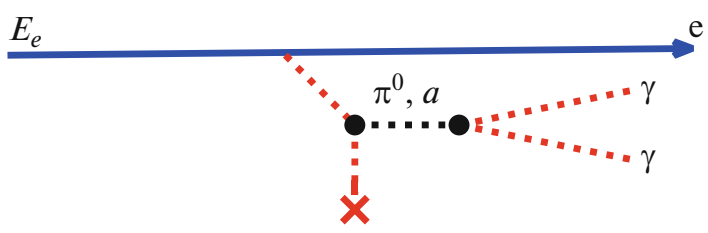

Fig. 12. (a) Production of hypothetical Dark photon or X17 production, (b) gamma conversion into two gammas due to Primakoff effect with axion-like particles.

(a)

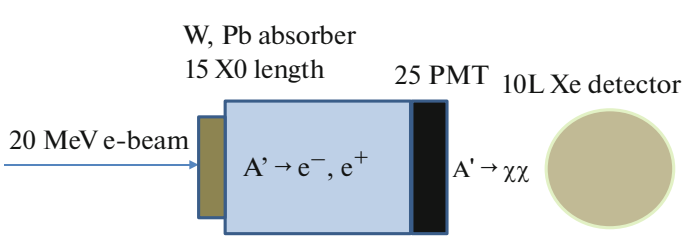

(b)

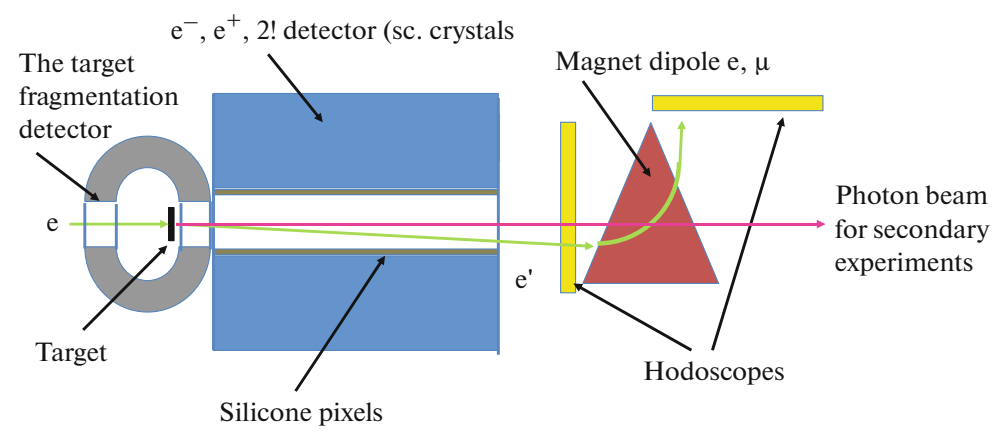

Fig. 13. (a) An initial setup configuration, (b) an advanced case.

look for their manifestations at the current accelerators [24, 25]. Another point of interest is related with recently observed $\mathrm{e}^{+} \mathrm{e}^{-}$-pair anomaly excess in the decay spectra of excited ${ }^{8} \mathrm{Be}$ and ${ }^{4} \mathrm{He}$ nuclei [26], which can be explained by new boson (X17) beyond $\mathrm{SM}$ with the mass near 16.7 MeV. Other low-mass particles beyond the SM sector are axion-like bosons, which should be important for understanding of CPviolation origin (Fig 12).

Available data from various experiments set rather stringent limits on the coupling constant values, e.g., for dark photons and X17-boson, see, e.g., the latest upper limits obtained by NA64 Collaboration with high energy electron beam (100 GeV) at CERN [27].

We propose here a setup, which can be installed at LINAC-200 to explore for possible candidates of Dark sector and other particles beyond the SM with $\mathrm{e}^{+} \mathrm{e}^{-}$ and $\gamma$-spectra in electron-nucleus collisions. At the initial stage it will be configured as a simple version (Fig. 13a), which can be upgraded later for an advanced configuration (Fig. 13b).

In the initial stage a simple setup configuration Fig. 13a can be used in beam dump experiment. The setup consists of $\mathrm{W}$ or $\mathrm{Pb}$ absorber (dump) and fast scintillator calorimeter with PMT. It can be supplied with Xenon ( $10 \mathrm{~L})$ detector (like developed prototype in MEPHI) to register light weakly interacting particles (neutralino, etc.) after decay of dark photon.

The beam energy $(\sim 20 \mathrm{MeV})$ for beam dump configuration should be below nuclear giant resonance excitation to avoid nuclear recoil fragments. For advanced case (Fig. 13b), with higher electron beam energy it should be required that transferred momentum should be below the giant resonance excitation. The advanced setup case, which includes fine electromagnetic calorimeter, silicon pixel detector, dipole magnet, etc., is capable for precision measurements in addition to $\mathrm{e}^{+} \mathrm{e}^{-}$spectra also of $\gamma \gamma$-spectra from $\gamma$-conversion via Primakoff effect. The advanced configuration in Fig. 13b allows to use the electron beam for secondary experiments.

Our estimates show that with high intensity $(\geq 100 \mu \mathrm{A})$ electron beam at energy $20-200 \mathrm{MeV}$ one can improve the current limits on new hypothetical particle production in mass range $1-20 \mathrm{MeV}$.

\section{Task 13}

Influence of electron beam parameters (pulse duration, pulse repetition rate, etc.) on irradiation efficiency of DNA/porphyrin complexes. Electron beam therapy is one of the efficient methods in treatment of surface tumors, such as skin cancer and limb diseases. Fast 
electrons ionize the DNA molecule, causing damage. There exist single-strand and double-strand breaks, DNA-DNA or DNA-protein cross-links [28]. Photodynamic therapy based on porphyrins in combination with electron beam therapy may increase the cancer treatment efficiency [29-31]. Electron beam therapy is performed at a medical linear accelerator. When electrons are required, an X-ray target is removed out of the beam, and the electron beam is collimated using an applicator or additional collimating inset manufactured from an alloy with low melting temperature. An electron beam has a finite range, after which irradiation dose drops abruptly. Therefore, deeper healthy tissues are intact. The penetration depth is adjusted by choosing an appropriate energy. Porphyrins are the well known compounds possessing high degree of conjugation, they may contain a central atom of metal, such as iron, magnesium, etc. Porphyrins have been studied at many laboratories for application as photosensibilizing agents in medicine [31].

The mechanism of DNA damage by electrons (especially low energy electrons) are well known. At the same time, the investigation of radiation damage on the molecular level in the presence of such substances as porphyrins, cis-platinum, remains topical. We studied DNA damage in the presence of a different amount of porphyrins (both metal-less and $\mathrm{Fe}, \mathrm{Cu}$ containing porphyrins) caused by $3-4 \mathrm{MeV}$ electron beam as a function of irradiation dose in vitro [32].

It is proposed to study DNA damage by electron beams of various energies with controllable beam parameters in the presence of antitumor agents cisplatinum and different metal porphyrins. This will provide detailed understanding of the impact of beam parameters on possible enhancing effects of irradiation with the substances of interest. LINAC-200 beams will provide data for different irradiation doses with higher precision than those available at present.

\section{Task 14}

Investigation of parametric $\mathrm{X}$-ray, transition and Smith-Purcell radiations in the presence of acoustic vibrations. Under certain conditions, the medium may essentially influence the characteristics of the highenergy electromagnetic processes. At high energies, the formation length exceeds the interatomic spacing, the interference effects from all atoms within this length are important. In crystals, where the atoms are arranged in ordered rows, the cross sections of the high-energy electromagnetic processes can change essentially compared with the corresponding quantities for a single atom. In particular, the coherent bremsstrahlung and coherent electron-positron pair production by photons in crystals have been widely investigated. The presence of a medium also gives rise to new types of radiation processes. Examples of the latter are Cherenkov, parametric X-ray (PXR), transition, and diffraction radiations. All these types of radi- ation arise due to the polarization of a medium by the field of moving charges. These radiation processes are widely used for generation of intense monochromatic radiation in various wavelength ranges, for identification of charged particles, for diagnostics of particle beams, and for investigation of the properties of matter and surfaces. From the point of view of controlling the parameters of the radiation processes in a medium, it is of interest to investigate the influence of external fields, such as acoustic waves, temperature gradient etc., on the corresponding characteristics. The studies of enhancement of radiation processes for charged particles interacting with media (in particular, with crystals) in the presence of ultrasonic vibrations were carried out at the Institute of Applied Problems of Physics, NAS RA. The examination of such processes as PXR [33], channeling radiation [34], transition radiation [35], bremsstrahlung of high-energy electrons [36], and electron-positron pair creation by high-energy photons [37] showed that the external fields can substantially change the spectral and angular characteristics of radiation intensity. The SmithPurcell and transition radiation on surface waves, excited on planar interfaces between two media, were investigated in [38, 39].

We propose to study PXR and transition radiation from relativistic electrons in piezoelectric crystals in the presence of acoustic superlattices in order to create an intense narrow-directed and monochromatic sources of short-wave radiation with space-time controlled parameters. To achieve these goals, experiments will be conducted for observation of PXR and transition radiation in various samples in the presence of ultrasonic vibrations. The parameters of these vibrations resulting in the maximum amplification and controllability will be specified. As the second class of problems, we aim to investigate the SmithPurcell radiation from bunches flying over a surface wave excited on a planar interface. The experimental setup here is similar to that for observation of the Smith-Purcell radiation on diffraction grating. An additional arrangement will be installed to excite surface waves that will allow one to control the parameters of the generated periodic structure on the same sample. The third class of problems will include the experimental investigation of the Cherenkov radiation in cylindrical waveguides loaded by a periodic structure [40] and emission of surface polaritons in cylindrical waveguides by charged particles [41].

\section{INSTALLATIONS AND EQUIPMENT}

Below are the experimental setups developed and/or operated by the project team members which underlie the present expertize of the project team.

- setup "Röntgen-1", Lebedev Physical Institute (7 MeV electrons); 
$100 \mathrm{keV}$ );

- Belgorod State University (electrons up to

- Institute for High Energy Physics, Protvino (protons up to $50 \mathrm{GeV}$, carbon ions up to $350 \mathrm{MeV} /$ nucleon);

- accelerator CLEAR, CERN (electrons up to $210 \mathrm{MeV}$ )-the setup is being prepared to be installed at CLEAR.

The project team possesses a unique material and hardware infrastructure for preparation of the research in the framework of the project. KEK, Tomsk Polytechnic University, Lebedev Physical Institute, and Belgorod State University have test facilities with accelerated electron beams where the project equipment will be tested before performing experiments at LINAC-200. In particular, these centers provide accelerated electron beams with an energy of 8,5 , and $7 \mathrm{MeV}$. Belgorod State University has a specialized unit certified for development and testing of generating $\mathrm{X}$-ray sources with an energy of up to $150 \mathrm{keV}$ (License no. 77.99.15.002.L.000093.10.19 of 02.10.2019). Most team members have clearance to work with generating $\mathrm{X}$ ray sources. It should also be noted that Belgorod State University has a production laboratory for manufacturing vacuum chamber and vacuum-compatible robotized systems which will be used in creation of experimental stations in the framework of the project.

\section{CONCLUSIONS}

Various research tasks of the new project FLAP for applied and fundamental physics are briefly discussed. These tasks can be accomplished using a high intensity linear electron accelerator facility with energy range from tens of $\mathrm{MeV}$ to a few hundred $\mathrm{MeV}$. It is planned to start experimental studies shortly at the LINAC-200 facility (JINR).

\section{REFERENCES}

1. M. Shevelev, A. Aryshev, N. Terunuma, and J. Urakawa, "Generation of a femtosecond electron microbunch train from a photocathode using twofold Michelson interferometer," Phys. Rev. Accel. Beams 20, 103401 (2017).

2. M. Fukuda et al., "Generation of multi-bunch beam with beam loading compensation by using RF amplitude modulation in Laser Undulator Compact X-ray (LUCX)," in Proceedings of the 6th International Particle Accelerator Conference IPAC'15, Richmond, VA, USA, May 2015, pp. 1576-1578.

https://doi.org/10.18429/JACoW-IPAC2015-TUPWA065

3. V. V. Soboleva et al., Nucl. Instrum. Methods Phys. Res., Sect. B 402, 182-184 (2017).

4. S. M. Young et al., Phys. Rev. Lett. 108, 140405 (2012).

5. Qinsheng Wang et al., Nano Lett. 17, 834-841 (2017).

6. T. Nebiki et al., J. Vacuum Sci. Technol. A 21, 1671 (2003).
7. K. A. Vokhmyanina et al., Nucl. Instrum. Methods Phys. Res., Sect. B 355, 307-310 (2015).

8. R. M. Nazhmudinov, A. S. Kubankin, P. V. Karataev, et al., "A multi-wirescanner test setup utilizing characteristic X-rays for charged particle and photon beam diagnostics," J. Instrum. 13, 12012 (2018).

9. K. A. Vokhmyanina, A. Aryshev, A. S. Kubankin, I. A. Kishin, N. Terunuma, and J. Urakawa, "Prebunched relativistic electron beam focusing by dielectric capillary," in Proceedings of the 13th International Symposium on Radiation from Relativistic Electrons in Periodic Structures, September 16-20, 2019.

10. A. Kubankin, V. Likhachev, N. Nasonov, A. Rakitjansky, and P. Zhukova, "Cherenkov effect and parametric X-rays," Nucl. Instrum. Methods Phys. Res., Sect. B 252, 124-130 (2006).

11. R. Kieffer, L. Bartnik, M. Bergamaschi, V. V. Bleko, M. Billing, L. Bobb, J. Conway, M.Forster, P. Karataev, A. S. Konkov, R. O. Jones, T. Lefevre, J. S. Markova, S. Mazzoni, Y. Padilla Fuentes, A. P. Potylitsyn, J. Shanks, and S. Wang, "Direct observation of incoherent Cherenkov diffraction radiation in the visible range," Phys. Rev. Lett. 121, 054802 (2018).

12. A. Curcio, M. Bergamaschi, R. Corsini, W. Farabolini, D. Gamba, L. Garol, R. Kieffer, T. Lefevre, S. Mazzoni, K. Fedorov, J. Gardelle, A. Gilardi, P. Karataev, K. Lekomtsev, T. Pacey, Y. Saveliev, A. Potylitsyn, and E. Senes, "Non-invasive bunch length measurements exploiting Cherenkov diffraction radiation," Phys. Rev. Accel. Beams 23, 022802 (2020).

13. G. Naumenko, A. Potylitsyn, M. Shevelev, P. Karataev, M. Shipulya, and V. Bleko, "Monochromatic coherent transition and diffraction radiation from a relativistic electron bunch train,” J. Instrum. 13, C04007 (2018).

14. G. Naumenko, A. Potylitsyn, P. Karataev, M. Shipulya, and V. Bleko, "Spectrum of coherent transition radiation generated by modulated electron beam," JETP Lett. 106, 127-130 (2017).

15. A. Aryshev, S. Araki, M. Fukuda, N. Terunuma, J. Urakawa, P. Karataev, G. Naumenko, A. Potylitsyn, L. Sukhikh, D. Verigin, and K. Sakaue, "Observation of the stimulated coherent diffraction radiation in an open resonator at LUCX facility," Nucl. Instrum. Methods Phys. Res., Sect. A 763, 424-432 (2014).

16. A. A. Krasnykh, I. A. Miloichikova, G. A. Naumenko, Yu. M. Cherepennikov, and S. G. Stuchebrov, "Reconstruction of the electron beam flux density distribution in the transverse plane based on the multi-angle wire scanning,” Nauch. Vedom. Belgor. Univ., Fiz. Mat. 50, 323328 (2018).

17. A. A. Baldin, A. I. Berlev, A. N. Fedorov, and N. V. Kudashkin, "Detector based on microchannel plates for monitoring space-time parameters of circulating beams of Nuclotron,” Phys. Part. Nucl. Lett. 2, 121-126 (2014).

18. A. A. Baldin, A. I. Berlev, S. E. Vasil'ev, A. V. Vishnevsky, et al., "Monitoring of extracted beams of Nuclotron accelerator complex for experiments 'energy + transmutation'," JINR Rapid Commun., No. 2 (JINR, Dubna, 2016). 
19. A. A. Baldin, G. A. Feofilov, P. Har'yuzov, and F. F. Valiev, "Fast beam-beam collisions monitor for experiments at NICA," Nucl. Instrum. Methods Phys. Res., Sect. A 958, 162154 (2019).

20. J. Adam, A. A. Baldin, M. Baznat, et al., "Secondary particle distributions in an extended uranium target under irradiation by proton, deuteron, and carbon beams", Nucl. Instrum. Methods Phys. Res., Sect. A 872, 87-92 (2017).

21. Yu. B. Bazarov, M. A. Karpov, E. V. Khaldeev, et al., "Results of registration of light phenomena in a shockloaded quartz single crystal," in Proceedings of the 35th International Conference on Equations of State for Matter ELBRUS 2020, Cheget, Russian Federation, March 1-6, 2020.

22. M. A. Karpov, N. A. Kleopova, and S. A. Zatolokin, "Registrator of frame X-ray images with direct transformation and an exposure from 5 ns in a range from 0.01 to $300 \mathrm{keV}$," in Proceedings of the 5th International Conference on Laser, Plasma Studies and Technologies, February 12-15, 2019, Moscow.

23. M. Battaglieri et al., in Summary of Workshop on U. S. Cosmic Visions: New Ideas in Dark Matter, 2017, arXiv: 1707.04591

24. D. Banerjee et al. (NA64 Collab.), Phys. Rev. Lett. 118, 011802 (2017).

25. G. B. Franklin et al. (APEX Coll.), EPJ Web of Conf. 142, 01015 (2017).

26. A. J. Krasznahorkay et al., Phys. Rev. Lett. 116, 042501 (2016).

27. D. Banerjee et al. (NA64 Collab.), Phys. Rev. D 101, 071101 (2020).

28. P. Swiderek, Angew. Chem. Int. Ed. 45, 4056 (2006).

29. M. B. Vrouenraets, G. W. Visser, G. B. Snow, and G. A. van Dongen, Anticancer Res. 23 (1B), 505 (2003).

30. B. C. Wilson, Can. J. Gastroenterol. 16, 393 (2002).

31. A. E. O'Connor, W. M. Gallagher, and A. T. Byrne, Photochem. Photobiol. 85, 1053 (2009).

32. V. M. Tsakanov, L. R. Aloyan, Y. B. Dalyan, et al., "AREAL low energy electron beam applications in life and materials sciences," Nucl. Instrum. Methods Phys. Res., Sect. A 829, 248-253 (2016).

33. A. R. Mkrtchyan et al., Phys. Status Solidi A 92, 361 (1985); A. R. Mkrtchyan, H. A. Aslanyan, A. H. Mkrtchian, R. H. Gasparian, and G. M. Garibian, Phys. Lett. A 152, 297 (1991).
34. A. R. Mkrtchyan, R. A. Gasparyan, and R. G. Gabrielyan, Phys. Lett. A 115, 410 (1986); Phys. Lett. A 126 528 (1988); L. Sh. Grigoryan et al., Nucl. Instrum. Methods Phys. Res., Sect. B 173, 13 (2001); Nucl. Instrum. Methods Phys. Res., Sect. B 173, 184 (2001); L. Sh. Grigoryan, A. H. Mkrtchyan, H. F. Khachatryan, V. U. Tonoyan, and W. Wagner, Nucl. Instrum. Methods Phys. Res., Sect. B 201, 25 (2003); W. Wagner, B. Azadegan, L. Sh. Grigoryan, and J. Pawelke, Europhys. Lett. 78, 56004 (2007); B. Azadegan, L. Sh. Grigoryan, J. Pawelke, and W. Wagner, J. Phys. B 41, 235101 (2008).

35. L. Sh. Grigoryan, A. H. Mkrtchyan, and A. A. Saharian, Nucl. Instrum. Methods Phys. Res., Sect. B 145, 197 (1998); A. R. Mkrtchyan, V. V. Parazian, and A. A. Saharian, Mod. Phys. Lett. B 27, 2693 (2010); A. R. Mkrtchyan, V. V. Parazian, and A. A. Saharian, Int. J. Mod. Phys. B 26, 1250036 (2012); A. R. Mkrtchyan, V. V. Parazian, and A. A. Saharian, J. Instrum. 13, C01032 (2018).

36. A. A. Saharian, A. R. Mkrtchyan, V. V. Parazian, and L. Sh. Grigoryan, Mod. Phys. Lett. A 19, 99 (2004); A. R. Mkrtchyan, A. A. Saharian, and V. V. Parazian, Mod. Phys. Lett. B 23, 2573 (2009).

37. A. R. Mkrtchyan, A. A. Saharian, L. Sh. Grigoryan, and B. V. Khachatryan, Mod. Phys. Lett. A 17, 2571 (2002); A. R. Mkrtchyan, A. A. Saharian, and V. V. Parazian, Mod. Phys. Lett. B 20, 1617 (2006).

38. A. R. Mkrtchyan, L. Sh. Grigoryan, A. A. Saharian, and A. N. Didenko, Acustica 75, 1984 (1991); A. A. Saharian, A. R. Mkrtchyan, L. A. Gevorgian, L. Sh. Grigoryan, and B. V. Khachatryan, Nucl. Instrum. Methods Phys. Res. Sect. B 173, 211 (2001).

39. A. R. Mkrtchyan, A. P. Potylitsyn, V. R. Kocharyan, and A. A. Saharian, Phys. Rev. E 93, 022117 (2016).

40. L. Sh. Grigoryan, H. F. Khachatryan, and S. R. Arzumanyan, Nuovo Cim. C 34, 317 (2011); L. Sh. Grigoryan, A. R. Mkrtchyan, H. F. Khachatryan, S. R. Arzumanyan, and W. Wagner, J. Phys.: Conf. Ser. 357, 012004 (2012); A. R. Mkrtchyan et al., J. Instrum. 15, C06019 (2020).

41. A. S. Kotanjyan, A. R. Mkrtchyan, A. A. Sahariana, and V. Kh. Kotanjyan, J. Instrum. 13, C01016 (2018); A. A. Saharian, A. S. Kotanjyany, L. Sh. Grigoryan, H. F. Khachatryan, and V. Kh. Kotanjyan, Int. J. Mod. Phys. B 34, 2050065 (2020); A. A. Saharian, L. Sh. Grigoryan, A. Kh. Grigorian, H. F. Khachatryan, and A. S. Kotanjyan, Phys. Rev. A 102, 063517 (2020). 